\title{
Proactive versus reactive task-set inhibition: Evidence from flanker compatibility effects
}

\author{
David Kuhns And Mei-Ching Lien \\ Oregon State University, Corvallis, Oregon \\ AND \\ ERIC RUTHRUFF \\ University of New Mexico, Albuquerque, New Mexico
}

\begin{abstract}
Two converging tests were used to determine whether people proactively inhibit recently performed tasks when switching to new tasks. A task-cuing paradigm was used. In each trial, the relevant stimulus was accompanied by flankers belonging either to the task performed on the immediately preceding trial (lag 1) or a more distant trial (lag 2+). If the just-performed task is inhibited when switching to another task, and this inhibition declines across trials, then flanker interference should be smaller with lag 1 flankers than with lag $2+$ flankers. Experiment 1, following the methods of Hübner, Dreisbach, Haider, and Kluwe (2003), failed to confirm this prediction. The prediction was confirmed in Experiment 2, however, using a design modified to provide greater incentives for task-set inhibition. The results provide evidence that inhibition can be applied proactively, to reduce the ability of an abandoned task to interfere with the performance of other tasks.
\end{abstract}

To better understand how the mind controls itself, researchers have utilized the task-switching paradigm. In one variant, known as the alternating-runs paradigm, participants receive a repeating task sequence, such as AABB throughout a block (e.g., Rogers \& Monsell, 1995). In another popular variant, the task sequence is unpredictable but a task cue (often an explicit task name) indicates which task should be performed next (e.g., Meiran, 1996). Regardless of the paradigm, performance is slower and less accurate following a task switch. This switch cost is especially large when each stimulus is associated with two active tasks (bivalent), rather than just one (univalent).

Switch costs have been attributed to the carryover of a previously activated task set (called task-set inertia; see, e.g., Allport, Styles, \& Hsieh, 1994), and the time needed to configure a new task (called task-set reconfiguration; see, e.g., Rogers \& Monsell, 1995; Ruthruff, Remington, \& Johnston, 2001). Another line of debate concerns whether switch costs reflect an inability to complete taskset preparation in advance of the stimulus (see, e.g., Lien, Ruthruff, Remington, \& Johnston, 2005) or occasional failures to engage in preparation (see, e.g., De Jong, 2000). In the present study, we focus on another mechanism hypothesized to contribute to task-switch costs-namely, task-set inhibition (see, e.g., Mayr \& Keele, 2000). Such inhibition would facilitate the immediate switch to a new task, but might impede a subsequent switch back to the inhibited task.

\section{$n-2$ Repetition Costs As an Index of Task-Set Inhibition}

To look for evidence of task-set inhibition, Mayr and Keele (2000) examined task switch performance as a function of how recently the task was performed (see also e.g., Arbuthnott, 2005; Schuch \& Koch, 2003). The critical prediction is that response time (RT) should be longer for more recently performed tasks, because the inhibition of that task would have had less opportunity to decay (but see Gade \& Koch, 2005). They compared performance on the third trial of an ABA task sequence (where inhibition of Task $A$ is recent) versus the third trial of a CBA task sequence (where inhibition of Task $A$ is less recent). Hereafter, we refer to these conditions as the $n-2$ repetition and $n-2$ switch conditions, respectively. Many studies have reported an $n-2$ repetition cost: slower responses in the $n-2$ repetition condition than the $n-2$ switch condition. The cost is somewhat counterintuitive, in that repetition generally produces a benefit, but makes perfect sense in light of task-set inhibition theory.

\section{Is Inhibition Applied Proactively or Reactively?}

Mayr and Keele (2000) proposed that inhibition is applied to a task proactively, just after it has been performed, to reduce interference from that task on a subsequent switch trial. In support of this claim, they reported $n-2$ repetition costs in an ABA sequence even when there was no Task A distractor to inhibit (reactively) in the intervening trial.

M.-C. Lien, mei.lien@oregonstate.edu 
Other studies, however, have argued for reactive task-set inhibition, where inhibition is applied in response to a conflict detected between a relevant task and an irrelevant task. Goschke (2000), for instance, found that task-switch RT was longer following trials in which the relevant and irrelevant tasks suggested incompatible responses rather than compatible responses (but see Lien, Ruthruff, \& Kuhns, 2006). This finding suggests that response conflict increases task inhibition. Because Goschke used only two tasks, however, it is unclear whether incompatible flankers lead to increased reactive inhibition of the irrelevant task or increased activation of the relevant task (which might then cause extra interference on the next trial). Further evidence for reactive inhibition was provided by Schuch and Koch (2003), who found $n-2$ repetition costs in an $\mathrm{ABA}$ sequence when the intervening trial was a go trial, but not when it was a no-go trial. This finding suggests that inhibition is applied reactively, during performance of the intervening task, rather than proactively. Taken together, these studies question whether task-set inhibition really is proactive, and suggest a need for converging lines of evidence.

\section{Flanker Effects as a Converging Index of Proactive Task-Set Inhibition}

$n-2$ repetition costs measure a negative "side-effect" of proactive task-set inhibition: difficulty switching back to an inhibited task. However, it is also possible to measure the direct, positive effects of proactive task-set inhibition: reduced interference from that task on the next trial. Specifically, irrelevant flankers should produce less interference when they belong to the most recently performed task (just inhibited) compared to a less recently performed task.

Hübner, Dreisbach, Haider, and Kluwe (2003) were the first to explicitly test this prediction. In their study, the three tasks (odd/even, consonant/vowel, and straight/ curved line) used unique sets of response keys. Task sequences were unpredictable and consisted of both task repetitions and task switches. Half of the task-switch trials were preceded by a color cue, indicating the required task, and half were not. Even without a task cue, participants could determine which task to perform because the target was always presented in the center, between two identical flanking stimuli. The critical manipulation was whether the flankers were drawn from the most recently performed task (trial $n-1$ ) or the less recently performed task (e.g., trial $n-2$ or $n-3$ ). For ease of discussion, we refer to flankers from the task performed on trial $n-1$ as lag 1 flankers, and flankers from trial $n-2$ or earlier as lag $2+$ flankers.

On switch trials, task-set inhibition theory predicts less interference from flankers belonging to the preceding task set (i.e., lag 1 flankers), because that task was most recently inhibited, than from lag $2+$ flankers. Hübner et al. (2003) confirmed this prediction when an advance task cue was provided. The opposite result was obtained when no cue was provided or when the cue indicated that the task would switch without indicating what the new task would be. They concluded that task-set inhibition is ap- plied only when participants prepare to switch to a specific task.

Hübner et al.'s (2003) flanker paradigm appears to provide fairly direct evidence that proactive task-set inhibition is doing exactly what it is hypothesized to be doing: reducing the ability of a just-performed task to interfere with the switch to another task. The empirical evidence, however, is still less than convincing. This effect has (to our knowledge) been reported in only one published study. Furthermore, that study found the effect predicted by inhibition theory in only one of four conditions (i.e., with an advance cue for a specific task). Even in that condition, Hübner et al. did not find the $n-2$ repetition costs obtained by Mayr and Keele (2000).

It is discouraging that the two measures of inhibition (flanker effects and $n-2$ repetition costs) disagreed. We suspect, however, that several aspects of Hübner et al.'s (2003) design discouraged participants from strongly inhibiting tasks, leading to mixed results. First, the center target stimulus (e.g., a digit between two identical letters) in Hübner et al.'s study was itself univalent - associated with only one of the three tasks. As pointed out by Mayr and Keele (2000), inhibition is most likely to occur when task sets must be activated endogenously, rather than exogenously. Second, each task used a distinct pair of response keys, potentially reducing competition between tasks and the need for task-set inhibition. Thus, to provide direct evidence for proactive taskset inhibit, one would need to modify Hübner et al.'s (2003) method to increase the incentives for task-set inhibition.

\section{The Present Study}

The purpose of the present study was to look for converging evidence for proactive task-set inhibition using Hübner et al.'s (2003) flanker paradigm. One critical test involved examining performance as a function of whether the flanker belongs to the more recently performed task (lag 1) or the less recently performed task (lag $2+$ ). If the just-performed task is inhibited proactively, and this inhibition decreases as task lag increases, then flanker interference should be smaller with lag 1 flankers than with lag $2+$ flankers.

As a converging measure of task-set inhibition, we also calculated $n-2$ repetition costs. Task-set inhibition predicts slower responses for more recently performed tasks (i.e., ABA) than for less recently performed tasks (i.e., CBA), because inhibition of that task would have had less time to decay (Mayr \& Keele, 2000).

\section{EXPERIMENT 1}

Experiment 1 replicated Hübner et al.'s (2003) design, but with a few simplifications. As in Hübner et al.'s study, we presented a target between two identical flankers and used three different response sets for the three tasks (accordingly, the flanker and target always suggested different responses). Hübner et al. mixed task-switch and task-repetition trials, but the data analyses focused on task-switch trials only. We therefore included task-switch trials only, as in most previous studies of task-set inhibition. Also, because Hübner et al. found evidence for 


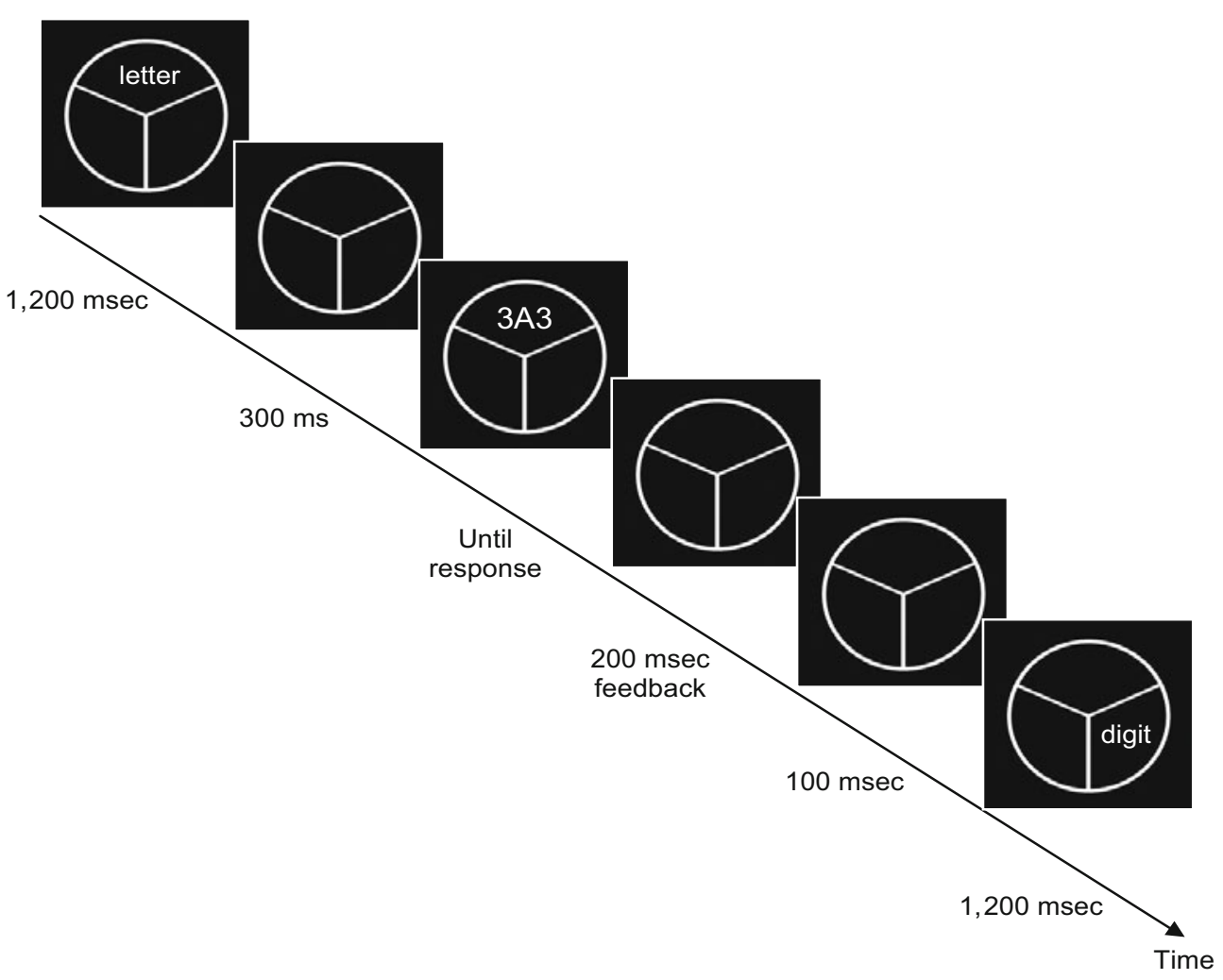

Figure 1. An example of the display and event sequence in Experiment 1.

task-set inhibition only when a cue specified the identity of the upcoming task, we used this condition only. These changes not only simplified the design, but also allowed us to collect more data in the conditions of interest.

\section{Method}

Participants. Sixty-four undergraduates from Oregon State University and the University of New Mexico participated in exchange for extra course credit.

Apparatus and Stimuli. Stimuli were presented on a 19-in. monitor. A white circular frame $8 \mathrm{~cm}$ in diameter, divided into three equal segments (see Figure 1), was presented in the screen center. On each trial, three stimuli $(0.8 \mathrm{~cm}$ wide $\times 0.9 \mathrm{~cm}$ tall $)$ appeared inside one of these segments. At an average viewing distance of $55 \mathrm{~cm}$, each stimulus subtended a visual angle of $0.92^{\circ} \times 1.04^{\circ}$.

Design and Procedure. On each trial, participants performed either a digit task, letter task, or symbol task by pressing keys on a standard English keyboard. For the digit task, participants pressed the " $Z$ " key for odd digits $(1,3,7,9)$ and the " $M$ " key for even digits $(2,4,6,8)$. For the letter task, participants pressed the " $X$ " key for consonants $(\mathrm{G}, \mathrm{K}, \mathrm{M}, \mathrm{R})$ and the " $\mathrm{N}$ " key for vowels (A, E, I, U). For the symbol task, participants pressed the "C" key for curved symbols $(\S, @, \beta, ?)$ and the "B" key for straight symbols $(\#,=,<$, !). Participants pressed the "Z," "X," and " $C$ " keys with the ring, middle, and index fingers of their left hand, respectively, and the "M," "N," and "B" keys with the ring, middle, and index fingers of their right hand, respectively.

Participants performed only task switch trials. The first trial of each block appeared in the top segment. Every trial began with a task cue, consisting of the word "letter," "digit," or "symbol" in the center of the segment for 1,200 msec. After a 300 msec blank period, the stimuli appeared until the participant responded. Auditory feedback $(200 \mathrm{msec})$ followed incorrect responses and silence
(200 msec) followed correct responses. The cue for the next trial appeared $100 \mathrm{msec}$ later in the segment located immediately clockwise from the previous one (see Figure 1); the stimuli continued rotating around the circle, clockwise, throughout each block. Thus, the location of the upcoming stimulus was always known in advance.

In each trial, the three stimuli were presented simultaneously in a row, with the target between two identical flankers (see Figure 1). Target identity was chosen randomly, with the restriction that each category (odd, even, consonant, vowel, curve, or straight) be selected equally often within each block. Flanker identity was selected randomly from the task recently performed (lag 1 ) or the less recently performed task (lag $2+),{ }^{1}$ with the restriction that the flanker and target never belong to the same task. Table 1 lists all possible types of task sequences.

Participants performed three practice blocks. The first practice block contained 12 trials. The second and third practice blocks, and the following 6 experimental blocks, contained 96 trials each. Participants received RT and accuracy feedback after each block. The experiment lasted approximately $45 \mathrm{~min}$.

\section{Results}

Trials were excluded from analysis if RT was less than $100 \mathrm{msec}$ or greater than $4,000 \mathrm{msec}(0.36 \%$ of trials $)$. Trials were also excluded if the current flanker stimulus (e.g., the digit " 4 ") was the previous target stimulus, which might lead to stimulus-specific "negative priming"; this exclusion allows us to examine task-level inhibition unconfounded with stimulus-level inhibition. Also excluded from RT analyses were error trials and trials following error trials.

Mean RT and proportion of error (PE), including all types of task sequences, were analyzed as a function of 
Table 1

All Possible Sequences for the Case Where the Second and Third Tasks Were Task $B$ and Task A, Respectively

\begin{tabular}{ccc}
\hline \multirow{2}{*}{ Flanker Lag } & \multicolumn{2}{c}{$n-2$ Task Transition } \\
\cline { 2 - 3 } & $\begin{array}{c}\text { Repetition } \\
(\text { e.g., A-B-A) }\end{array}$ & $\begin{array}{c}\text { Switch } \\
(\text { e.g., } \mathrm{C}-\mathrm{B}-\mathrm{A})\end{array}$ \\
\hline Lag 1 & $\mathrm{Ac}-\mathrm{Ba}-\mathrm{Ab}^{*}$ & $\mathrm{Ca}-\mathrm{Ba}-\mathrm{Ab}{ }^{*}$ \\
& $\mathrm{Ab}-\mathrm{Ba}-\mathrm{Ab}^{*}$ & $\mathrm{Cb}-\mathrm{Ba}-\mathrm{Ab}^{*}$ \\
& $\mathrm{Ac}-\mathrm{Bc}-\mathrm{Ab}$ & $\mathrm{Ca}-\mathrm{Bc}-\mathrm{Ab}$ \\
& $\mathrm{Ab}-\mathrm{Bc}-\mathrm{Ab}$ & $\mathrm{Cb}-\mathrm{Bc}-\mathrm{Ab}$ \\
$\mathrm{Aag} 2+$ & $\mathrm{Ac}-\mathrm{Ba}-\mathrm{Ac} c^{*}$ & $\mathrm{Ca}-\mathrm{Ba}-\mathrm{Ac}{ }^{*}$ \\
& $\mathrm{Ab}-\mathrm{Ba}-\mathrm{Ac} c^{*}$ & $\mathrm{Cb}-\mathrm{Ba}-\mathrm{Ac}{ }^{*}$ \\
& $\mathrm{Ac}-\mathrm{Bc}-\mathrm{Ac}$ & $\mathrm{Ca}-\mathrm{Bc}-\mathrm{Ac}$ \\
& $\mathrm{Ab}-\mathrm{Bc}-\mathrm{Ac}$ & $\mathrm{Cb}-\mathrm{Bc}-\mathrm{Ac}$ \\
\hline
\end{tabular}

Note-There were also analogous sequences, not shown, that ended with Tasks $\mathrm{C}-\mathrm{A}, \mathrm{A}-\mathrm{B}, \mathrm{C}-\mathrm{B}, \mathrm{A}-\mathrm{C}$, and $\mathrm{B}-\mathrm{C}$. The task sequences were classified according to whether the third task (Task A in this example) was a repetition or a switch with respect to the first task (i.e., $n-2$ task transition) and whether the flanker for the third task was from the most recently performed task (lag 1 ) or the less recent performed task (lag 2+). The uppercase letters indicate the relevant task, and the lowercase letters indicate the flanker task. "Task sequences in which the third task matched the previous flanker task.

$n-2$ task transition (repetition or switch) and flanker lag (lag 1 or lag $2+$ ). ${ }^{2}$ Table 2 shows mean RT and PE for each condition.

RT was 18 msec slower in the $n-2$ repetition condition $(820 \mathrm{msec})$ than the $n-2$ switch condition ( $802 \mathrm{msec})$ $\left[F(1,63)=12.61, M S_{\mathrm{e}}=3,130, p<.001\right]$. In addition, overall RT was longer with lag 1 flankers $(817 \mathrm{msec})$ than with lag $2+$ flankers $(806 \mathrm{msec})\left[F(1,63)=7.35, M S_{\mathrm{e}}=\right.$ $2,039, p<.01]$. The interaction between $n-2$ task transition and flanker lag was not significant $[F(1,63)<1.0]$.

For PE, only the main effect of $n-2$ task transition was significant $\left[F(1,63)=14.46, M S_{\mathrm{e}}=0.0004, p<.001\right]$. Consistent with the RT data, PE was .041 in the $n-2$ repetition condition and .034 in the $n-2$ switch condition.

\section{Discussion}

Experiment 1 produced a modest $n-2$ repetition cost (18 msec), consistent with task inhibition that declines gradually across trials (Mayr \& Keele, 2000). As in Hübner et al. (2003), we also examined whether flanker interference was smaller with lag 1 flankers than with lag $2+$ flankers, as predicted by proactive task-set inhibition. Despite the design similarities, the effect actually went in the

Table 2

Mean Response Times (RTs, in Milliseconds) and Proportions of Errors (PEs) in Experiment 1 As a Function of n-2 Task Transition and Flanker Lag

\begin{tabular}{|c|c|c|c|c|c|c|}
\hline \multirow[b]{3}{*}{ Flanker Lag } & \multicolumn{4}{|c|}{$n-2$ Task Transition } & & \\
\hline & \multicolumn{2}{|c|}{$\begin{array}{l}\text { Repetition } \\
\text { (e.g., ABA) } \\
\end{array}$} & \multicolumn{2}{|c|}{$\begin{array}{c}\text { Switch } \\
\text { (e.g., CBA) }\end{array}$} & \multicolumn{2}{|c|}{ Average } \\
\hline & RT & $\mathrm{PE}$ & RT & $\mathrm{PE}$ & RT & $\mathrm{PE}$ \\
\hline Lag 1 & 826 & .041 & 807 & .035 & 817 & .038 \\
\hline $\operatorname{Lag} 2+$ & 814 & .041 & 797 & .034 & 806 & .038 \\
\hline Average & 820 & .041 & 802 & .035 & & \\
\hline
\end{tabular}

Note-There was no lag 2 flanker in the $n-2$ repetition condition and no lag 3 flanker in the $n-2$ switch condition. opposite direction, by $11 \mathrm{msec}(p<.05)$. This finding suggests that the previously performed task was the most active, not the most inhibited.

The data analyses reported above included all possible types of task sequences. As shown in Table 1, however, the different flanker lag conditions naturally allowed different relationships between the tasks and flankers for trial $n$ and trial $n-1$. The lag $2+$ flanker condition, for example, included sequences where the current flanker task was also the previous flanker task (e.g., the task sequence Bc-Ac), but the lag 1 flanker condition did not. We therefore conducted an additional data analyses restricted to sequences in which the current target was the previous flanker (see Table 1), which occurred both for lag 1 flankers and lag $2+$ flankers. This restricted analysis supported the main analysis reported above. The $n-2$ repetition cost was $26 \mathrm{msec}$ for RT $\left[F(1,63)=14.50, M S_{\mathrm{e}}=3,015, p<\right.$ $.0003]$, and 0.008 for $\mathrm{PE}\left[F(1,63)=9.32, M S_{\mathrm{e}}=0.0004\right.$, $p<.01]$. In addition, RT was $13 \mathrm{msec}$ longer with the lag 1 flanker than with lag $2+$ flanker, a marginally significant difference $\left[F(1,63)=3.75, M S_{\mathrm{e}}=2,809, p=\right.$ $.0574]$.

In summary, we were unable to obtain converging evidence for proactive task-set inhibition under conditions similar to those of Hübner et al. (2003). Interestingly, Hübner et al. found evidence of inhibition based on flanker effects, but not based on $n-2$ repetition costs (essentially the opposite of our results). Despite these differences, both studies failed to produce converging evidence for task-set inhibition.

\section{EXPERIMENT 2}

As discussed above, there are at least two reasons to suspect that the conditions used in Hübner et al. (2003), and the present Experiment 1, did not promote proactive task-set inhibition. First, the target was always presented in the center of the display, allowing participants to focus spatial attention on that stimulus. The perception of the stimulus itself might have been sufficient to drive task preparation (bottom-up), reducing the need for inhibition of irrelevant tasks. Note that the stimuli in Experiment 1 were associated only with their own task sets (i.e., univalent stimuli). Second, each task used a distinct pair of response keys, potentially reducing competition between tasks.

The goal of Experiment 2 was to further increase the incentive for task-set inhibition. First, we placed one target and one flanker side-by-side in each trial, with their relative location determined randomly. Because participants did not know where the target would appear, they would need to either activate the relevant task and/or inhibit the irrelevant task. Second, we used the same pair of response keys for each of the three tasks. Thus, unlike Experiment 1, the target and the flanker in each trial could activate the same response key (the compatible condition) or different response keys (the incompatible condition).

Although we could just examine the flanker lag effect on incompatible trials, as in Experiment 1, we focused on a more specific measure of the task-set activation- 

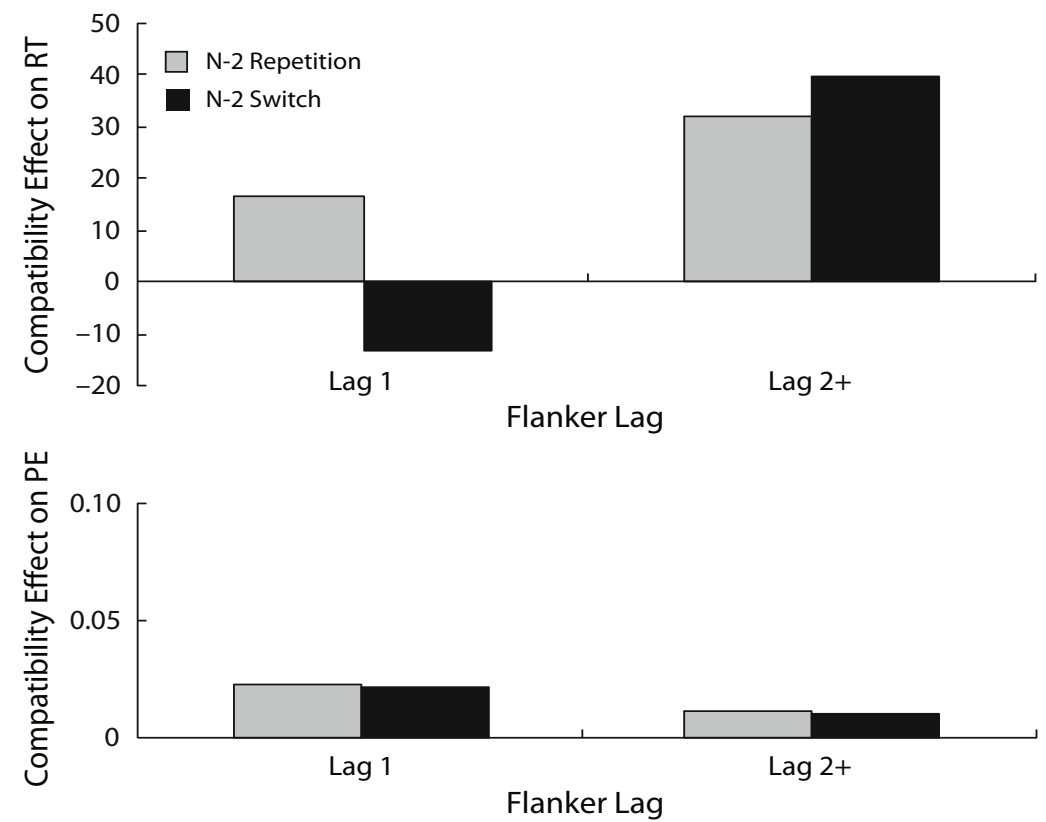

Figure 2. Target/flanker response compatibility effects (incompatible minus compatible) on response time (RT, in milliseconds) and proportion of errors $(\mathrm{PE})$ in Experiment 2 as a function of flanker lag (lag 1 or lag $2+$ ) and $n-2$ task transition (repetition or switch).

namely, the flanker compatibility effect (defined as the RT difference between compatible and incompatible trials). If the just-performed task is inhibited when switching to another task, and this interference declines across trials, one would expect to see smaller flanker compatibility effects when the current flanker was drawn from the most recently performed task (lag 1) than when it was drawn from the less recently performed task (lag $2+)$.

\section{Method}

The methods were the same as in Experiment 1, except as noted below.

Participants. There were 56 new participants.

Apparatus, Stimuli, and Procedure. Only one target and one flanker were presented in each trial; their relative locations (left vs. right) were determined randomly. Participants pressed the " $Z$ " key with their left index finger for odd digits, consonants, and curved symbols and pressed the " $M$ " key with their right index finger for even digits, vowels, and straight symbols.

\section{Results}

The data analyses were similar to those of Experiment 1. Application of the RT cutoffs eliminated approximately $0.32 \%$ of trials. Data were analyzed as a function of $n-2$ task transition (repetition or switch), flanker lag (lag 1 or lag $2+$ ), and target/flanker compatibility (compatible or incompatible). Table 3 shows mean RT and PE data for each condition.

For RT, there was a significant $n-2$ repetition cost of $24 \mathrm{msec}\left[F(1,55)=15.08, M S_{\mathrm{e}}=4,307, p<.001\right]$. The overall target/flanker compatibility effect $(18 \mathrm{msec})$ on RT was statistically significant $\left[F(1,55)=7.55, M S_{\mathrm{e}}=\right.$ $5,045, p<.01]$. Mean RT was similar for both lag 1 and lag $2+$ flankers $[F(1,55)<1.0]$. Most importantly, however, flanker lag interacted significantly with compatibility $\left[F(1,55)=8.34, M S_{\mathrm{e}}=3,900, p<.01\right]$; the compatibility effect was only $1 \mathrm{msec}$ for lag 1 flankers, but was $35 \mathrm{msec}$ for lag $2+$ flankers. Focusing on incompatible trials only (similar to the analysis of Experiment 1), RT was significantly shorter with lag 1 flankers $(899 \mathrm{msec})$ than with lag $2+$ flankers $(916 \mathrm{msec})[F(1,55)=5.54$, $\left.M S_{\mathrm{e}}=6,268, p<.05\right]$.

For PE, the main effect of compatibility was significant $\left[F(1,55)=43.44, M S_{\mathrm{e}}=0.00075, p<.001\right]$, as well as its interaction with flanker lag $\left[F(1,55)=4.85, M S_{\mathrm{e}}=\right.$ $0.00069, p<.05]$. The compatibility effect was 0.022 for lag 1 flankers and 0.012 for lag $2+$ flankers (See Figure 2). No other effects were significant.

Table 3

Mean Response Times (RTs, in Milliseconds) and Proportions of Errors (PEs) in Experiment 2 As a Function of $\boldsymbol{n}-2$ Task Transition, Flanker Lag, and Target/Flanker Compatibility

\begin{tabular}{|c|c|c|c|c|c|c|}
\hline \multirow[b]{3}{*}{ Flanker Lag } & \multicolumn{4}{|c|}{$n-2$ Task Transition } & & \\
\hline & \multicolumn{2}{|c|}{$\begin{array}{l}\text { Repetition } \\
\text { (e.g., ABA) }\end{array}$} & \multicolumn{2}{|c|}{$\begin{array}{c}\text { Switch } \\
\text { (e.g., CBA) }\end{array}$} & \multicolumn{2}{|c|}{ Average } \\
\hline & RT & $\mathrm{PE}$ & RT & $\mathrm{PE}$ & RT & $\mathrm{PE}$ \\
\hline \multicolumn{7}{|l|}{ Lag 1} \\
\hline Compatible & 904 & .030 & 891 & .035 & 898 & .033 \\
\hline Incompatible & 920 & .054 & 878 & .056 & 899 & .055 \\
\hline \multicolumn{7}{|l|}{$\operatorname{Lag} 2+$} \\
\hline Compatible & 892 & .035 & 869 & .036 & 880 & .035 \\
\hline Incompatible & 924 & .046 & 908 & .047 & 916 & .047 \\
\hline
\end{tabular}

Note-There was no lag 2 flanker in the $n-2$ repetition condition and no lag 3 flanker in the $n-2$ switch condition. 


\section{Discussion}

To further promote task-set inhibition, we presented one target and one flanker in random positions and used the same response keys for all tasks. As in Experiment 1, a significant $n-2$ repetition cost (24 msec) was observed, replicating Mayr and Keele's (2000) findings. Unlike Experiment 1 , the flanker lag effects also indicated task-set inhibition: The compatibility effect was smaller for lag 1 flankers than for lag $2+$ flankers. These findings converge on the conclusion that task-set inhibition facilitates the immediate switch to a new task by proactively inhibiting the task performed on the preceding trial. Although we do not know which of our design modifications (making the stimuli more bivalent and/or making the responses more bivalent) was primarily responsible for different results, we conclude that the change reflects increased need for task-set inhibition.

As in Experiment 1, we conducted follow-up analyses restricted to the conditions common to both flanker lags, in which the current target was the previous flanker (see Table 1). These analyses revealed similar results as the main analyses: The $n-2$ repetition cost was $12 \mathrm{msec}$ $\left[F(1,55)=4.78, M S_{\mathrm{e}}=9,784, p<.05\right]$, and the compatibility effect was smaller for lag 1 flankers $(6 \mathrm{msec})$ than for lag $2+$ flankers $(42 \mathrm{msec})\left[F(1,55)=4.61, M S_{\mathrm{e}}=\right.$ $8,036, p<.05]$.

\section{GENERAL DISCUSSION}

Task-set inhibition theory predicts that recently performed (and thus recently inhibited) tasks will be easy to ignore when they become irrelevant, but will be difficult to return to when they once again become relevant. The present study tested both of these predictions at the same time. Experiment 1, using the tasks of Hübner et al. (2003), failed to confirm these predictions. Although participants had difficulty switching to recently performed tasks (there was a significant $n-2$ repetition cost), they actually had more trouble ignoring recently performed tasks than less recently performed tasks. These findings raise the possibility that inhibition was not applied proactively (see also Lien et al., 2006; Philipp \& Koch, 2006).

We argued that certain aspects of Hübner et al.'s (2003) design (univalent stimuli and univalent responses), adopted in the present Experiment 1, discourage strong task-set inhibition. Experiment 2, therefore, increased the incentives for task-set inhibition by randomizing the location of the target and flanker and using the same two response keys for each task (as in most previous studies of task-set inhibition). Under these more demanding conditions, both predictions of proactive task-set inhibition theory were confirmed. We observed $n-2$ repetition costs as well as smaller flanker compatibility effects for lag 1 flankers than for lag $2+$ flankers. Thus, this study provides important converging evidence, using a combination of two different measures (flanker and $n-2$ repetition) with different assumptions, that task-set inhibition does occur when it is needed most.

\section{Proactive Inhibition versus Reactive Inhibition}

The small compatibility effects produced by flankers belonging to the just-performed task (lag 1) follow naturally from proactive inhibition, but not reactive inhibition. An even more direct test of proactive versus reactive inhibition is to compare the trials where the current flanker task was from the immediately preceding target task (as in $\mathrm{Cb}-\mathrm{Ac}$ ) and trials where the current flanker task was the immediately preceding flanker task (as in Bc-Ac). Proactive inhibition asserts that the most strongly inhibited task is the immediately preceding target task; flanker effects should therefore be smallest with flankers from the immediately preceding target task. Reactive inhibition, however, asserts that the most strongly inhibited task is the immediately preceding flanker task; flanker effects should therefore be smallest with flankers from the immediately preceding flanker task.

The data from Experiment 1 were more consistent with reactive inhibition: RT was $11 \mathrm{msec}$ faster when the current flanker was the previous flanker rather than the previous target $\left[F(1,63)=4.40, M S_{\mathrm{e}}=1,978, p<.05\right]$. Under conditions designed to more strongly promote task-set inhibition (Experiment 2), however, the data supported the proactive inhibition prediction: Flanker compatibility effects were smaller, albeit nonsignificantly, when the current flanker was from the immediately preceding target task $(-6 \mathrm{msec})$, rather than from the immediately preceding flanker task $(17 \mathrm{msec})\left[F(1,55)=1.98, M S_{\mathrm{e}}=8,072\right.$, $p=.1648]$. Reactive inhibition might be especially strong following a trial in which the target and flanker were incompatible (Goschke, 2000). However, data analyses including only trials following incompatible trials showed no evidence for reactive inhibition: Flanker compatibility effects in Experiment 2 were still significantly smaller when the current flanker task was the previous target task than when it was the previous flanker task $[F(1,55)=6.11$, $\left.M S_{\mathrm{e}}=9,009, p<.05\right]$.

A plausible speculation is that, when task switching is relatively easy (as in Experiment 1), inhibition is weaker and mostly reactive. But as task-switching difficulty increases (as in Experiment 2), inhibition becomes stronger and more proactive. Further research is needed to directly test this speculation.

\section{Conclusion}

Previous studies have suggested that cognitive control in task switching involves inhibition of the just-performed task set. We found support for task-set inhibition (at least under certain favorable conditions) using two converging operations with different assumptions. Importantly, these results provide direct evidence that, in addition to any reactive inhibition, inhibition can also be applied proactively.

\section{AUTHOR NOTE}

This research was supported by funding from the Oregon Space Grant Consortium Graduate Fellowship to D.K. and the Oregon State University Research Incentive Programs to M.-C.L. Correspondence concerning this article should be addressed to M.-C. Lien, Department of Psychology, Oregon State University, Corvallis, OR 97331 (e-mail: mei.lien@oregonstate.edu). 


\section{REFERENCES}

Allport, A., Styles, E. A., \& Hsieh, S. (1994). Shifting intentional set: Exploring the dynamic control of tasks. In C. Umiltà and M. Moscovitch (Eds.), Attention and performance $X V$ : Conscious and nonconscious information processing (pp. 421-452). Cambridge, MA: MIT Press.

Arbuthnott, K. D. (2005). The influence of cue type on backward inhibition. Journal of Experimental Psychology: Learning, Memory, \& Cognition, 31, 1030-1042.

De Jong, R. (2000). An intention-activation account of residual switch costs. In S. Monsell \& J. Driver (Eds.), Attention and performance XVIII: Control of cognitive processes (pp. 357-376). Cambridge, MA MIT Press.

GADE, M., \& KocH, I. (2005). Linking inhibition to activation in the control of task sequences. Psychonomic Bulletin \& Review, 12, 530-534.

GoschKe, T. (2000). Intentional reconfiguration and involuntary persistence in task set switching. In S. Monsell \& J. Driver (Eds.), Attention and performance XVIII: Control of cognitive processes (pp. 331-355). Cambridge, MA: MIT Press.

Hübner, R., Dreisbach, G., Haider, H., \& Kluwe, R. H. (2003). Backward inhibition as a means of sequential task-set control: Evidence for reduction of task competition. Journal of Experimental Psychology: Learning Memory, \& Cognition, 29, 289-297.

Lien, M.-C., Ruthruff, E., \& Kuhns, D. (2006). On the difficulty of task switching: Assessing the role of task-set inhibition. Psychonomic Bulletin \& Review, 13, 530-535.

Lien, M.-C., Ruthruff, E., Remington, R. W., \& Johnston, J. C. (2005). On the limits of advance preparation for a task switch: Do people prepare all the task some of the time or some of the task all the time? Journal of Experimental Psychology: Human Perception \& Performance, 31, 299-315.

Lien, M.-C., Schweickert, R., \& Proctor, R. W. (2003). Taskswitching and response correspondence in the psychological refractory period paradigm. Journal of Experimental Psychology: Human Perception \& Performance, 29, 692-712.

Mayr, U., \& Keele, S. W. (2000). Changing internal constraints on action: The role of backward inhibition. Journal of Experimental Psychology: General, 129, 4-26.

MeIRAN, N. (1996). Reconfiguration of processing mode prior to task performance. Journal of Experimental Psychology: Learning, Memory, \& Cognition, 22, 1423-1442.

Philipp, A. M., \& KoCH, I. (2006). Task inhibition and task repetition in task switching. European Journal of Cognitive Psychology, 18, 624639.

Rogers, R. D., \& Monsell, S. (1995). Costs of a predictable switch between simple cognitive tasks. Journal of Experimental Psychology: General, 124, 207-231.

Ruthruff, E., Remington, R. W., \& Johnston, J. C. (2001). Switching between simple cognitive tasks: The interaction of top-down and bottom-up factors. Journal of Experimental Psychology: Human Perception \& Performance, 27, 1404-1419.

ScHUCH, S., \& KoCH, I. (2003). The role of response selection for inhibition of task sets in task shifting. Journal of Experimental Psychology: Human Perception \& Performance, 29, 92-105.

\section{NOTES}

1. The present experimental design generated two different types of task sequences: CBA $(n-2$ switch) and ABA $(n-2$ repetition). Note that the flanker for the third trial (Task A) in the CBA sequence could be from lag 1 or 2 only. However, the flanker for the third trial in the ABA sequence could be either from lag 1, 3, or longer. Because there are relatively few trials at longer lags, we collapsed the data across trials with lags of 2 or longer (lag $2+$ ).

2. In Experiment 1, only task-switch trials were used, with different response keys for each task. Thus, the target and flanker were always associated with different response keys (incompatible). However, the spatial configuration of the keys for each hand (left, middle, and right) could produce spatial correspondence effects (i.e., shorter RT when the target and the flanker activate spatially corresponding response keys). Nevertheless, the corresponding trials are relatively rare $(1 / 4)$, reducing statistical power. Furthermore, previous studies have shown negligible response correspondence effects for task switch trials (see Lien, Schweickert, \& Proctor, 2003). Accordingly, we followed Hübner et al. (2003) and emphasized predictions for overall RT in Experiment 1.

(Manuscript received July 21, 2006; revision accepted for publication November 29, 2006.) 